\title{
Depression and Generalized Anxiety disorders among Chronically Ill Middle and Older Adults in Saudi Arabia
}

\author{
Alshimaa Mohsen Mohamed Lotfy ${ }^{1}$, Bedour Saleh Dugim Alharbi², \\ Wafa Saleh dugim Alharbi ${ }^{3} \&$ Eman Elsayed Abd-Ellatif ${ }^{4}$ \\ ${ }^{1}$ Lecturer in Department of Public Health and Community Medicine Faculty of Medicine, Beni-Suef University, \\ Egypt \\ ${ }^{2}$ Medical Bachelor \& Bachelor of Surgery (MBBC), Egypt \\ ${ }^{3}$ Specialist Nurse in Alshifa Hospital, Egypt \\ ${ }^{4}$ Department of Public Health \& Community Medicine, Faculty of Medicine, Mansoura University, Egypt \\ Correspondence: Eman Elsayed Abd-Ellatif, Department of Public Health \& Community Medicine, Faculty of \\ Medicine, Mansoura University, Egypt
}

Received: September 27, 2021 Accepted: October 18, 2021 Online Published: November 2, 2021

doi:10.5539/gjhs.v13n12p25 URL: https://doi.org/10.5539/gjhs.v13n12p25

\begin{abstract}
Background: Depression and anxiety are common prevalent in patients with chronic disease, and Chronic illness are long-lasting conditions with persistent effects and complications. Depression and anxiety disorders are well known to have a negative but significant impact on the course, outcomes, long-term survival, and treatment efficacy of patients suffering from physical disease. Given the high prevalence and potential negative consequences of this type of comorbidity in elderly patients, there is an urgent need for this study to be conducted due to the scarcity of statistics on the topic among middle and older adults in the Al-Qassim region. On the other hand, early identification of people who are more likely to experience psychological distress is critical in prevention and management strategies.
\end{abstract}

Objectives: To assess the severity of depression and anxiety, using validated self-report measures of middle-aged and older adults; and to determine the association between anxiety and depression levels and socio-demographic characteristics.

Methods: In February 2021 and for two months, a cross-sectional study of middle- aged and older adults from Al-Shifa hospital at Al-Qassim region was conducted with questionnaires on socio-demographic and clinical characteristics, and validated Generalized Anxiety Disorders (GAD-7), and Patient Health Questionnaire (PHQ-9) anxiety and depression questionnaires. Two hundreds middle- aged and older adults participants of Al-Shifa Hospital participated in this study and agreed to complete the questionnaire.

Results: $79 \%(\mathrm{n}=158)$ of participants had minimal to mild depression and $21 \%(\mathrm{n}=42)$ had moderate-to-severe depression; the average PHQ-9 score was $7.05(\mathrm{M}=7.05$; $\mathrm{SD}=4.4)$, while $81.5 \%(\mathrm{n}=163)$ of participants had minimal to mild anxiety and $18.5 \%(\mathrm{n}=37)$ had moderate-to-severe anxiety; the average GAD score was $6.6(\mathrm{M}$ $=6.6 ; \mathrm{SD}=4.3)$. The significant predictors of moderate-to-severe depression in the logistic regression analysis were: male sex (odds ratio (OR):0.0.272; confidence interval (CI):0.101-0.730) and psychiatric disorders (odds ratio (OR):13.131; confidence interval (CI):2.975-57.949). The significant predictors of moderate-to-severe anxiety in the logistic regression analysis were psychiatric disorders (odds ratio (OR):13.080; confidence interval (CI):3.261-52.464). There was a strong significant positive correlation between PHQ-9 score and GAD score.

Conclusion: The study group had higher prevalence of moderate and severe depression, which was significantly associated with female sex and psychiatric disorders; while the prevalence of moderate and severe anxiety was significantly associated with the presence of psychiatric disorders. Our findings reaffirm the importance of providing appropriate specialized interventions to promote the mental health of chronically ill elderly, with a focus on high-risk groups.

Keywords: elderly, chronically ill, Saudi Arabia, anxiety, depression, GAD-7 and PHQ-9 


\section{Introduction}

Population ageing is one of the most significant global demographic trends (Thapa, Visentin, Kornhaber, \& Cleary, 2020). Saudi Arabia demographic trends are changing. The kingdom follows the global increase in aging population. As a result, elderly population of those aged 60 and above is projected to increase from $3 \%$ in 2010 to $9.5 \%$ and $18.4 \%$ in 2035 and 2050, respectively (Khoja et al., 2018).

Ageing can increase the risk of mental health problems and subsequently the prevalence of mental health disorders has been observed to be higher among the older people. More than one fifth of older adults aged experience a mental disorder with depression, dementia and anxiety the most common(Thapa et al., 2020).

In recent decades, the global prevalence of depression and depressive symptoms has increased. In women, the lifetime prevalence of depression ranges from $20 \%$ to $25 \%$, while in men it ranges from $7 \%$ to $12 \%$. Depression is a significant predictor of quality of life and survival, accounting for roughly half of all psychiatric consultations and $12 \%$ of all hospital admissions. (Wang et al., 2017) A meta-analysis found the lifetime prevalence of depressive disorders for older adults was 16.5\% in Western countries (Reynolds, Pietrzak, El-Gabalawy, Mackenzie, \& Sareen, 2015).

In the general population, anxiety disorders are the most common type of psychiatric disorder. According to the Global Burden of Disease study, anxiety disorders account for approximately 26.8 million disability-adjusted life years and have an annual direct cost of $\$ 42.3$ billion. Generalized anxiety disorder (GAD) is characterized by persistent worry as well as a number of additional symptoms such as restlessness, muscle tension, and difficulty concentrating. It is a common and disabling condition in adults that can impair social and occupational functioning. Despite the fact that there is an effective treatment for GAD, only a small percentage of those affected receive it. This is due to the fact that anxiety disorders are frequently misdiagnosed and mismanaged by clinicians in primary care, which is frequently the first point of contact for those with mental health problems (Remes et al., 2018). The prevalence of anxiety disorder was $11.4 \%$ among US older adults (Reynolds et al., 2015).

Mental disorders in older adults contribute to increased mortality, higher suicide rates, cognitive disorders, with negative effects on well-being and quality of life. Older adults with mental disorders may experience physical health problems such as stroke and heart disease, increased disability and limited capacity to undertake activities of daily living, increased use of health and home care services, financial issues, and social isolation (Thapa et al., 2020).

The correlates of mental health conditions among older people may differ from younger cohorts, with differing levels of exposure and impacts. Older people are more likely to experience life stressors related to functional ability, cognition and mobility, chronic pain, fragility and bereavement. These factors may contribute to mental disorders such as depression, anxiety, stress, isolation, loneliness or psychological distress in older people (WHO, 2017).

Vink et al. (2008) provided a comprehensive review on the risk factors for depression and anxiety among older adults. Common risk factors for depression and anxiety were female gender, lack of social support, adverse life events, functional limitations, chronic health conditions, poor self-perceived health, personality traits, inadequate coping strategies and psychopathology (Vink, Aartsen, \& Schoevers, 2008).

In studies conducted in a variety of countries around the world, depressive and anxiety disorders have been repeatedly established as very common in primary care settings (Goldberg et al., 2017).

The coexistence of depression and generalized anxiety disorder (GAD) is a consistent finding across age groups. Comorbidity in adults is associated with a worse prognosis, greater functional disability, and higher service utilization than in people with a single disorder. Much less is known about the course and consequences of depression with coexisting GAD in the elderly (Schoevers, Deeg, Van Tilburg, \& Beekman, 2005).

Despite their prevalence and importance in primary care, as well as their significant contribution to disability, rates of identification and treatment remain very low, with less than half of depressive episodes correctly identified even in high-resource primary care settings (Goldberg et al., 2017).

Depression and anxiety disorders are well known to have a negative but significant impact on the course, outcomes, long-term survival, and treatment efficacy of patients suffering from physical disease (Guerrero \& Ramos, 2015).

Given the high prevalence and potential negative consequences of this type of comorbidity in elderly patients, there is an urgent need for this study to be conducted due to the scarcity of statistics on the topic among middle and older adults in the Al-Qassim region. On the other hand, early identification of people who are more likely to experience psychological distress is critical in prevention and management strategies. 
The aim of this study is to assess the levels of anxiety and depression in a sample of middle and older adults using validated self-report measures. Our secondary aim was to determine psychologically vulnerable groups by examining the relationship between anxiety and depression levels and socio-demographic characteristics.

\section{Material and Methods}

\subsection{Study Design \& Setting}

A cross-sectional study design was conducted among the elderly in Unayzah, Al-Qassim, Saudi Arabia from February to April, 2021.

\subsection{Study Participants}

Chronically ill Elderly attending Alshifa Hospital (geriatric hospital), Unayzah, Al-Qassim region were the target population. The study included both; male and female who agreed to participate, and those who refused to participate; or having any psychiatric disorders will be excluded from the study.

\subsection{Study Sample}

All chronically ill elderly selected from Al Shifa hospital in Unayzah; Al-Qassim region; agreed to participate. Between $1^{\text {st }}$ February 2021 until $1^{\text {st }}$ April 2021 were included in the study. Two hundreds middle- aged and older adults participants of Al-Shifa Hospital participated in this study and completed the questionnaire.

\subsection{Data Collection Method and Tools}

A structured interview questionnaire was administered to willing participants. The questionnaire divided into 3 parts was used to assess depression and generalized anxiety disorders among study participants at Al Qassim region, Saudi Arabia. A pilot study for 30 users at Al Shifa hospital were carried out before data collection to ensure clarity and easiness of the questionnaire. Asked every participant over the last 2 weeks, how often have you been bothered by any of the following problems?

Part 1: A 5-item question; Socio-demographic data of study participants included: age, gender, education level, nationality and having any chronic diseases.

Part 2: A 9-item question; using the validated Patient Health Questionnaire (PHQ-9) to assess depression severity. This is calculated by assigning scores of $0,1,2$, and 3 to the response categories, respectively, of "not at all," "several days," "more than half the days," and "nearly every day." GAD-7 total score for the seven items ranges from 0 to 27. It is measured by the 5-point Likert scale. Final response scores were identified as: Minimal depression (1-4); moderate depression (5-9); moderately severe depression (15-19); and severe depression (20-27) The psychometric properties of the PHQ-9 scores have been widely supported, and the reliability of the scale among previous studies was excellent $(\alpha=0.92)$.(Shevlin et al., n.d.)

Part 3: A 9-item question; using the validated Generalized Anxiety Disorders (GAD-7), This is calculated by assigning scores of $0,1,2$, and 3 to the response categories, respectively, of "not at all," "several days," "more than half the days," and "nearly every day." GAD-7 total score for the seven items ranges from 0 to 21 . It is measured by the 5-point Likert scale. Final response scores were identified as: minimal anxiety (0-4); mild anxiety (5-9); moderate anxiety 910-14) and severe anxiety (15-21). The GAD-7 has been shown to produce reliable and valid scores in community studies, 17 and the reliability in the current sample was high $(\alpha=0.94)$.(Hinz et al., 2016)

\subsection{Data Management and Analysis}

SPSS program version 25 (Statistical Package for Social science) for Windows. Mean, standard deviation (SD), median, and range were used to describe the quantitative variables (min-max). Numbers (number) and percentages (\%) were used to describe the qualitative variables. Chi-Squared test/fisher exact was used to test the relationship between categorical variables. Multivariable binary logistic regression analysis was performed to analyze the risk variables for the occurrence of depression and anxiety in the form of adjusted odds ratio and confidence interval for the odds ratio. The significance of the results was assessed in the term of P-value which was differentiated into: non-significant when P-value $>0.05$ and significant when P-value $\leq 0.05$. The correlation coefficient was used to determine the association between different variables. P-value $\leq 0.05$ reflected the significant level.

\subsection{Ethical Consideration}

Ethical approval was obtained from the Research, Ethical Committee of the General Directorate of Health, Al-Qassim Region of the Ministry of Health-Saudi Arabia (H-04-Q-001-1442-1140657 on -February 01-2021), and each participant gave written informed consent. This consent was included in the first section of the questionnaire. Participants were informed that the information collected from them would be kept confidential. 


\section{Result}

Out of the total 200 middle- aged and older adults participants of Al-Shifa Hospital participated in this study and completed the questionnaire. Their ages ranged from 45 to 80 years, with a mean of $60.35 \pm 7.8$ years. The proportion of Female study participants was $55 \%$. The vast majority the respondents were married ( $89 \%)$. In terms of educational level, $25 \%$ of the participants attended preparatory school, followed by $21 \%$ in primary school. The mean number of chronic diseases among the middle-aged and older adults participants was $2 \pm 1.5$. Eighty one percent of the study participants had chronic diseases, $62.5 \%$ of study participants had diabetes, $60 \%$ had hypertension, and $33.5 \%$ having CHD (Table 1 ).

Table 1. Socio-Demographic and Clinical Characteristics of the study Participants $(N=200)$

\begin{tabular}{|c|c|c|}
\hline Variables & $\mathbf{F}$ & $\%$ \\
\hline \multicolumn{3}{|l|}{ Sex } \\
\hline Females & 110 & 55.0 \\
\hline Males & 90 & 45.0 \\
\hline \multicolumn{3}{|l|}{ Marital status } \\
\hline Unmarried & 22 & 11.0 \\
\hline Married & 178 & 89.0 \\
\hline \multicolumn{3}{|l|}{ Education } \\
\hline Illiterate & 10 & 5.0 \\
\hline Read and write & 19 & 9.5 \\
\hline Primary & 43 & 21.5 \\
\hline Preparatory & 50 & 25.0 \\
\hline Secondary & 35 & $\mathbf{1 7 . 5}$ \\
\hline University & 34 & 17.0 \\
\hline Master & 5 & 2.5 \\
\hline $\mathrm{PhD}$ & 4 & 2.0 \\
\hline DM & 125 & 62.5 \\
\hline HTN & 121 & 60.5 \\
\hline CHD & 67 & 33.5 \\
\hline Renal & 22 & 11.0 \\
\hline Hematological & 13 & 6.5 \\
\hline Neurological & 7 & 3.5 \\
\hline Psychological & 13 & 6.5 \\
\hline Immunological & 7 & 3.5 \\
\hline Other chronic diseases & 21 & 10.5 \\
\hline Presence of chronic diseases & 162 & 81 \\
\hline Age $($ mean \pm SD $)$ & 60.35 & \\
\hline Median & 60 & \\
\hline Range (min-max) & $45-80$ & \\
\hline \multicolumn{3}{|l|}{ Number of chronic diseases } \\
\hline Mean \pm SD & \multicolumn{2}{|c|}{$2 \pm 1.5$} \\
\hline Min-max & \multicolumn{2}{|l|}{$0-6$} \\
\hline Median & \multicolumn{2}{|l|}{2} \\
\hline
\end{tabular}


Table 2. Frequency distribution PHQ-9 parameters among study participants (no=200)

\begin{tabular}{|c|c|c|c|c|}
\hline \multirow{2}{*}{$\begin{array}{l}\text { Depression PHQ-9 } \\
\text { (Over the last two weeks, how often have you been bothered by } \\
\text { the following problems) }\end{array}$} & Not at all & $\begin{array}{l}\text { Several } \\
\text { days }\end{array}$ & $\begin{array}{l}\text { More than } \\
\text { half the days }\end{array}$ & $\begin{array}{l}\text { Nearly } \\
\text { every day }\end{array}$ \\
\hline & $\mathbf{F}(\%)$ & $\mathbf{F}(\%)$ & $\mathbf{F}(\%)$ & $\mathbf{F}(\%)$ \\
\hline Q1-Little interest or pleasure in doing things & $48(24.0)$ & $131(65.5)$ & $15(7.5)$ & $6(3.0)$ \\
\hline Q2-Feeling down, depressed or hopeless & $40(20.0)$ & $125(62.5)$ & $25(12.5)$ & $10(5.0)$ \\
\hline Q3-Trouble falling or staying asleep, or sleeping too much & $46(23.0)$ & $113(56.5)$ & $34(17.0)$ & $7(3.5)$ \\
\hline Q4-Feeling tired or having little energy & $26(13.0)$ & $133(66.5)$ & $31(15.5)$ & $10(5.0)$ \\
\hline Q5-Poor appetite or overeating & $53(26.5)$ & $113(56.5)$ & $31(15.5)$ & $3(1.5)$ \\
\hline $\begin{array}{l}\text { Q6- Feeling bad about yourself or that you are a failure or have let } \\
\text { yourself or your family down }\end{array}$ & $106(53.0)$ & $71(35.5)$ & $16(8.0)$ & $7(3.5)$ \\
\hline $\begin{array}{l}\text { Q7- Trouble concentrating on things, such as reading the newspaper } \\
\text { or watching television }\end{array}$ & $86(43.0)$ & $92(46.0)$ & $15(7.5)$ & $7(3.5)$ \\
\hline $\begin{array}{l}\text { Q8- Moving or speaking so slowly that other people could have } \\
\text { noticed. Or the opposite being so fidgety or restless that you have } \\
\text { been moving around a lot more than usual }\end{array}$ & $129(64.5)$ & $57(28.5)$ & $11(5.5)$ & $3(1.5)$ \\
\hline $\begin{array}{l}\text { Q9- Thoughts that you would be better off dead, or of hurting } \\
\text { yourself }\end{array}$ & $150(75.0)$ & $43(21.5)$ & $4(2.0)$ & $3(1.5)$ \\
\hline
\end{tabular}

PHQ-9: Patient Health Questionnaire-9.

Regarding Generalized Anxiety Disorders (GAD-7), several days was the most common response to Q1 (56.5\%), Q2 (49.0\%), Q3 (52.5\%), Q4 (59.5\%), Q5 (54.0\%) and Q6 (61.0\%). 46.0\% reported that this was said, it Not at all for Q7 and also more than a third for Q2(35.5\%) and Q5 (32.5\%) (Table 3).

Table 3. Frequency distribution GAD-7 parameters among study participants (no=200)

\begin{tabular}{lllll}
\hline GAD-7 & $\begin{array}{l}\text { Not } \\
\text { (Over the last two weeks, how often have you been } \\
\text { bothered by the following problems) }\end{array}$ & $\begin{array}{l}\text { all } \\
\text { Qeveral } \\
\text { days }\end{array}$ & $\begin{array}{l}\text { More than half } \\
\text { the days }\end{array}$ & $\begin{array}{l}\text { Nearly every } \\
\text { day }\end{array}$ \\
\hline Q1. Feeling nervous, anxious, or on edge & $\mathbf{F}(\%)$ & $\mathbf{F}(\%)$ & $\mathbf{F}(\%)$ & $\mathbf{F}(\%)$ \\
\hline Q2. Not being able to stop or control worrying & $53(26.5)$ & $113(56.5)$ & $23(11.5)$ & $11(5.5)$ \\
\hline Q3. Worrying too much about different things & $71(35.5)$ & $98(49.0)$ & $21(10.5)$ & $10(5.0)$ \\
\hline Q4. Trouble relaxing & $45(22.5)$ & $105(52.5)$ & $34(17.0)$ & $16(8.0)$ \\
\hline Q5. Being so restless that it is hard to sit still & $52(26.0)$ & $119(59.5)$ & $20(10.0)$ & $9(4.5)$ \\
\hline Q6. Becoming easily annoyed or irritable & $65(32.5)$ & $108(54.0)$ & $18(9.0)$ & $9(4.5)$ \\
\hline Q7. Feeling afraid, as if something awful might happen & $92(46.0)$ & $75(37) 5$. & $17(8.5)$ & $19(9.5)$ \\
\hline
\end{tabular}

GAD-7: Generalized Anxiety Disorder-7.

The average PHQ-9 score for depression among study participants was 7.05 (mean $\pm \mathrm{SD}=7.05 \pm 4.4$ ), with mild depression accounting for just over half of participants (51.0\%), minimal depression (28.0\%), and moderate depression representing $13.0 \%$. The average GAD-7 score for anxiety was 6.6 (mean $\pm \mathrm{SD}=6.6 \pm 4.3$ ), with $49.5 \%$ of participants having mild anxiety, minimal anxiety $(32.0 \%$ ), and moderate anxiety $13.0 \%$ (Table 4 ). 
Table 4. Descriptive statistics of the total PHQ-9 and GAD-7 scores and severity among study participants $(\mathrm{n}=200)$

\begin{tabular}{ll}
\hline PHQ-9 & Values \\
\hline PHQ-9 score & \\
Mean \pm SD & $7.05 \pm 4.4$ \\
Min-max & $0-25$ \\
Median & 6 \\
PHQ-9 categories & $\underline{\mathrm{F}(\%)}$ \\
\hline Minimal depression & $56(28.0)$ \\
Mild depression & $102(51.0)$ \\
Moderate depression & $26(13.0)$ \\
Moderately severe depression & $14(7.0)$ \\
Severe depression & $2(1.0)$ \\
\hline GAD-7 & \\
\hline GAD -7 score & \\
Mean \pm SD & $6.6 \pm 4.3$ \\
Min-max & $0-21$ \\
Median & 6 \\
GAD -7 categories & $\mathrm{F}(\%)$ \\
Minimal anxiety & $64(32.0)$ \\
Mild anxiety & $99(49.5)$ \\
Moderate anxiety & $26(13.0)$ \\
Severe anxiety & $11(5.5)$ \\
\hline PHQ-9: Pater Helt
\end{tabular}

PHQ-9: Patient Health Questionnaire-9; GAD-7: Generalized Anxiety Disorder-7.

Table 5 shows that the severity of depression in the studied group is higher among those who are 60 years or older (62.0\% and $54.8 \%$, respectively), female $(53.8 \%$ and $59.5 \%$, respectively ), married $(90.5 \%$ and $83.3 \%$, respectively), have low to medium level of education $(77.8 \% \& 81.0 \%)$, have chronic diseases $(81.6 \% \& 78.6 \%)$ and have two chronic diseases or more $(63.7 \% \& 59.5 \%)$.

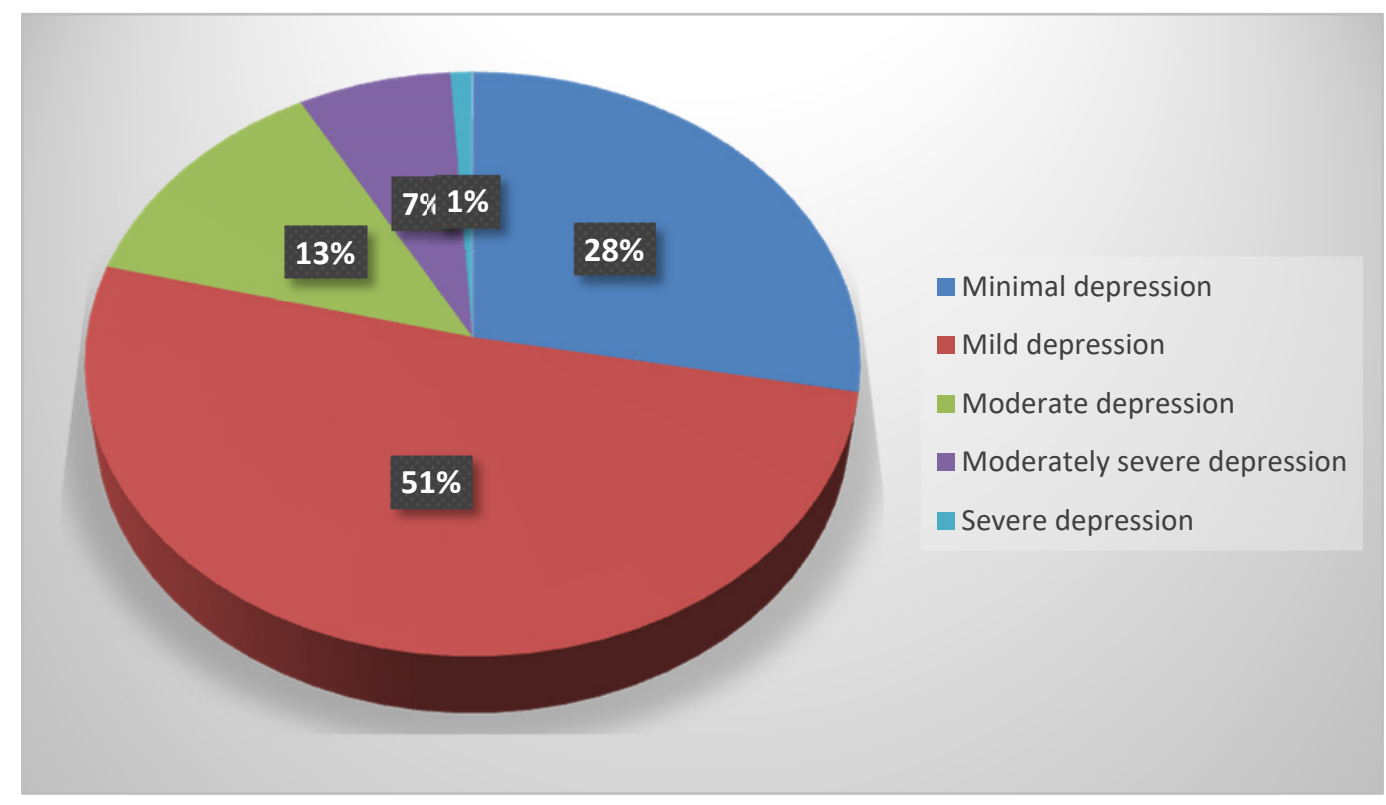

Figure 1. Showing different categories of depression among studied participants 


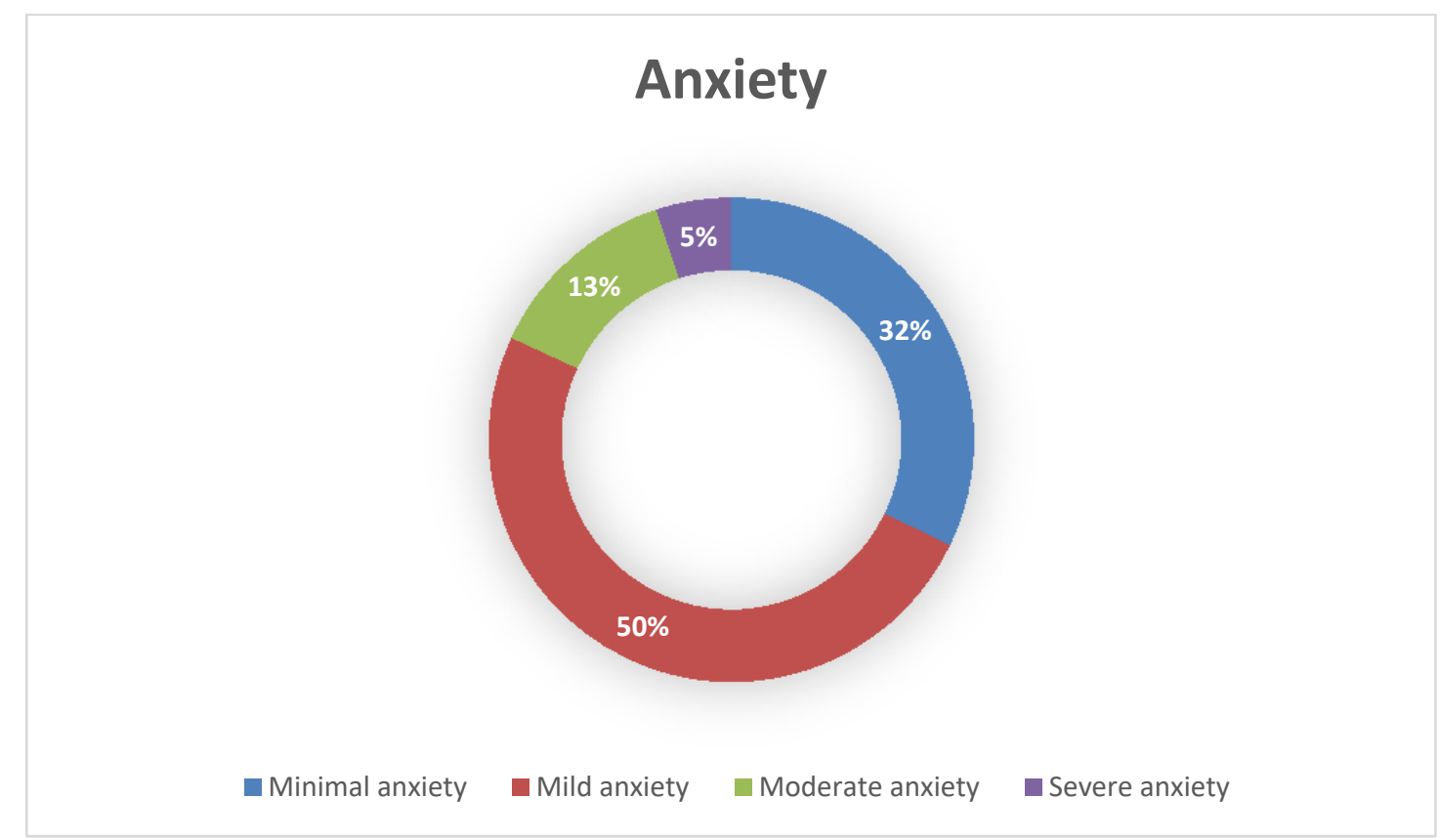

Figure 2. Showing different categories of anxiety among studied participants

Table 5. Comparison between depression severity regarding socio-Demographic and clinical Characteristics of the study Participants $(\mathrm{n}=200)$

\begin{tabular}{|c|c|c|c|c|c|}
\hline \multirow[t]{2}{*}{ Variables } & \multicolumn{2}{|c|}{$\begin{array}{l}\text { Minimal to mild } \\
(n=158)\end{array}$} & \multicolumn{2}{|c|}{$\begin{array}{l}\text { Moderate to severe } \\
(n=42)\end{array}$} & \multirow[t]{2}{*}{ p-value } \\
\hline & $\mathbf{F}$ & $\%$ & $\mathbf{F}$ & $\%$ & \\
\hline \multicolumn{6}{|l|}{ Age } \\
\hline$\leq 60$ years & 98 & $62.0 \%$ & 23 & $54.8 \%$ & 0.392 \\
\hline$>60$ years & 60 & $38.0 \%$ & 19 & $45.2 \%$ & \\
\hline \multicolumn{6}{|l|}{ Sex } \\
\hline Females & 85 & $53.8 \%$ & 25 & $59.5 \%$ & 0.507 \\
\hline Males & 73 & $46.2 \%$ & 17 & $40.5 \%$ & \\
\hline \multicolumn{6}{|l|}{ Marital status } \\
\hline Unmarried & 15 & $9.5 \%$ & 7 & $16.7 \%$ & 0.187 \\
\hline Married & 143 & $90.5 \%$ & 35 & $83.3 \%$ & \\
\hline \multicolumn{6}{|l|}{ Education Level } \\
\hline Illiterate & 7 & $4.4 \%$ & 3 & $7.1 \%$ & \\
\hline Read and write & 14 & $8.9 \%$ & 5 & $11.9 \%$ & \\
\hline Primary & 35 & $22.2 \%$ & 8 & $19.0 \%$ & \\
\hline Preparatory & 43 & $27.2 \%$ & 7 & $16.7 \%$ & 0.451 \\
\hline Secondary & 24 & $15.2 \%$ & 11 & $26.2 \%$ & \\
\hline University & 26 & $16.5 \%$ & 8 & $19.0 \%$ & \\
\hline Master & 5 & $3.2 \%$ & 0 & $0.0 \%$ & \\
\hline $\mathrm{PhD}$ & 4 & $2.5 \%$ & 0 & $0.0 \%$ & \\
\hline \multicolumn{6}{|l|}{ Education degree } \\
\hline Low to medium & 123 & $77.8 \%$ & 34 & $81.0 \%$ & 0.663 \\
\hline High & 35 & $22.2 \%$ & 8 & $19.0 \%$ & \\
\hline Chronic Diseases & & & & & \\
\hline
\end{tabular}




\begin{tabular}{|c|c|c|c|c|c|}
\hline \multicolumn{6}{|c|}{ Presence of chronic diseases } \\
\hline No & 29 & $18.4 \%$ & 9 & $21.4 \%$ & 0.652 \\
\hline Yes & 129 & $81.6 \%$ & 33 & $78.6 \%$ & \\
\hline \multicolumn{6}{|c|}{ Number of chronic diseases } \\
\hline$\leq 2$ diseases & 101 & $63.9 \%$ & 25 & $59.5 \%$ & 0.600 \\
\hline$>2$ diseases & 57 & $36.1 \%$ & 17 & $40.5 \%$ & \\
\hline \multicolumn{6}{|l|}{ DM } \\
\hline No & 58 & $36.7 \%$ & 17 & $40.5 \%$ & 0.654 \\
\hline Yes & 100 & $63.3 \%$ & 25 & $59.5 \%$ & \\
\hline \multicolumn{6}{|l|}{ HTN } \\
\hline No & 62 & $39.2 \%$ & 17 & $40.5 \%$ & 0.884 \\
\hline Yes & 96 & $60.8 \%$ & 25 & $59.5 \%$ & \\
\hline \multicolumn{6}{|l|}{ CHD } \\
\hline No & 103 & $65.2 \%$ & 30 & $71.4 \%$ & 0.446 \\
\hline Yes & 55 & $34.8 \%$ & 12 & $28.6 \%$ & \\
\hline \multicolumn{6}{|c|}{ Renal disorders } \\
\hline No & 140 & $88.6 \%$ & 38 & $90.5 \%$ & 0.731 \\
\hline Yes & 18 & $11.4 \%$ & 4 & $9.5 \%$ & \\
\hline \multicolumn{6}{|c|}{ Hematological disorders } \\
\hline No & 149 & $94.3 \%$ & 38 & $90.5 \%$ & 0.371 \\
\hline Yes & 9 & $5.7 \%$ & 4 & $9.5 \%$ & \\
\hline \multicolumn{6}{|c|}{ Neurological disorders } \\
\hline No & 154 & $97.5 \%$ & 39 & $92.9 \%$ & 0.148 \\
\hline Yes & 4 & $2.5 \%$ & 3 & $7.1 \%$ & \\
\hline \multicolumn{6}{|c|}{ Psychological disorders } \\
\hline No & 154 & $97.5 \%$ & 33 & $78.6 \%$ & $<0.001 *$ \\
\hline Yes & 4 & $2.5 \%$ & 9 & $21.4 \%$ & \\
\hline \multicolumn{6}{|c|}{ Immunological disorders } \\
\hline No & 155 & $98.1 \%$ & 38 & $90.5 \%$ & $0.017^{*}$ \\
\hline Yes & 3 & $1.9 \%$ & 4 & $9.5 \%$ & \\
\hline \multicolumn{6}{|c|}{ Others chronic diseases } \\
\hline No & 143 & $90.5 \%$ & 36 & $85.7 \%$ & 0.386 \\
\hline Yes & 15 & $9.5 \%$ & 6 & $14.3 \%$ & \\
\hline
\end{tabular}

$* p$-value $\leq 0.05$ is considered significant.

Low to medium education includes: Illiterate, Read and write, Primary, Preparatory and Secondary \& high education includes: University, Master and $\mathrm{PhD}$.

There is a statistically significant difference between the severity of depression and psychological disorders $(p<0.001)$, and immunological disorders $(p=0.017)$, but there was no significant difference between depression severity and DM, HTN, CHD, renal disorders, hematological disorders, neurological disorders, and other chronic diseases. After adjustment for age, sex, education, and the presence of a psychiatric disorders, Table 6 showed that male sex and the presence of a previous psychiatric disorder were associated with the occurrence of moderate to severe depression. Having a psychiatric disorder; increased the risk of depression by more than tenfold (OR, 95 percent CI for OR was $13.131,2.975$ to 57.949$)$. Being a male was found to decrease the risk of developing depression (OR, 95 percent CI for OR was $0.272,0.101$ to 0.730 ) (Table 6). 
Table 6. Multivariable binary logistic regression analysis for prediction of moderate to severe depression from different risk factors

\begin{tabular}{|c|c|c|c|c|c|}
\hline \multirow{2}{*}{ Independent variables } & \multirow{2}{*}{$\mathbf{B}$} & \multirow{2}{*}{ p-value } & \multirow{2}{*}{$\begin{array}{l}\text { Odds Ratio } \\
\text { (OR }\end{array}$} & \multicolumn{2}{|c|}{$95 \%$ CI for OR } \\
\hline & & & & Lower & Upper \\
\hline Old age $\geq 60$ years & 0.335 & 0.494 & 1.398 & 0.536 & 3.645 \\
\hline Male sex & -1.302 & $0.010^{*}$ & 0.272 & 0.101 & 0.730 \\
\hline High education & -0.183 & 0.774 & 0.833 & 0.238 & 2.914 \\
\hline Presence of psychiatric disorder & 2.575 & $0.001 *$ & 13.131 & 2.975 & 57.949 \\
\hline
\end{tabular}

$* P$-value $\leq 0.05$ is considered significant

$C I=$ confidence interval.

Table 7 illustrates that the severity of anxiety in the studied group was higher among those who 60 years or older (62.1\% and $62.2 \%$, respectively), female $(53.4 \%$ and $62.2 \%$, respectively), married $(88.3 \%$ and $83.3 \%$, respectively), low to medium level of education ( $78.5 \%$ and $78.40 \%$, respectively), suffer from chronic diseases $(82.2 \%$ and $75.7 \%$, respectively) and have chronic diseases or more $(63.8 \% \& 59.5 \%)$.

Table 7. Comparison between anxiety severity regarding to socio-Demographic and clinical Characteristics of the study Participants $(\mathrm{n}=200)$

\begin{tabular}{|c|c|c|c|c|c|}
\hline \multirow[t]{2}{*}{ Variables } & \multicolumn{2}{|c|}{$\begin{array}{l}\text { Minimal to mild } \\
(n=163)\end{array}$} & \multicolumn{2}{|c|}{$\begin{array}{l}\text { Moderate to severe } \\
(n=37)\end{array}$} & \multirow[t]{2}{*}{ p-value } \\
\hline & $\mathbf{F}$ & $\%$ & $\mathbf{F}$ & $\%$ & \\
\hline Age & & & & & 0.819 \\
\hline$\leq 60$ years & 98 & $60.1 \%$ & 23 & $62.2 \%$ & \\
\hline$>60$ years & 65 & $39.9 \%$ & 14 & $37.8 \%$ & \\
\hline Sex & & & & & 0.332 \\
\hline Females & 87 & $53.4 \%$ & 23 & $62.2 \%$ & \\
\hline Males & 76 & $46.6 \%$ & 14 & $37.8 \%$ & \\
\hline Marital status & & & & & 0.772 \\
\hline Unmarried & 19 & $11.7 \%$ & 3 & $8.1 \%$ & \\
\hline Married & 144 & $88.3 \%$ & 34 & $91.9 \%$ & \\
\hline Education level & & & & & 0.155 \\
\hline Illiterate & 5 & $3.1 \%$ & 5 & $13.5 \%$ & \\
\hline Read and write & 14 & $8.6 \%$ & 5 & $13.5 \%$ & \\
\hline Primary & 38 & $23.3 \%$ & 5 & $13.5 \%$ & \\
\hline Intermediate & 43 & $26.4 \%$ & 7 & $18.9 \%$ & \\
\hline Secondary & 28 & $17.2 \%$ & 7 & $18.9 \%$ & \\
\hline University & 26 & $16.0 \%$ & 8 & $21.6 \%$ & \\
\hline Master & 5 & $3.1 \%$ & 0 & $0.0 \%$ & \\
\hline $\mathrm{PhD}$ & 4 & $2.5 \%$ & 0 & $0.0 \%$ & \\
\hline Education level & & & & & 0.999 \\
\hline Low to medium & 128 & $78.5 \%$ & 29 & $78.4 \%$ & \\
\hline High & 35 & $21.5 \%$ & 8 & $21.6 \%$ & \\
\hline
\end{tabular}

Chronic diseases 


\begin{tabular}{|c|c|c|c|c|c|}
\hline No & 29 & $17.8 \%$ & 9 & $24.3 \%$ & \\
\hline Yes & 134 & $82.2 \%$ & 28 & $75.7 \%$ & \\
\hline Number of chronic diseases & & & & & 0.621 \\
\hline$\leq 2$ diseases & 104 & $63.8 \%$ & 22 & $59.5 \%$ & \\
\hline$>2$ diseases & 59 & $36.2 \%$ & 15 & $40.5 \%$ & \\
\hline DM & & & & & 0.054 \\
\hline No & 56 & $34.4 \%$ & 19 & $51.4 \%$ & \\
\hline Yes & 107 & $65.6 \%$ & 18 & $48.6 \%$ & \\
\hline HTN & & & & & 0.330 \\
\hline No & 67 & $41.1 \%$ & 12 & $32.4 \%$ & \\
\hline Yes & 96 & $58.9 \%$ & 25 & $67.6 \%$ & \\
\hline CHD & & & & & 0.879 \\
\hline No & 108 & $66.3 \%$ & 25 & $67.6 \%$ & \\
\hline Yes & 55 & $33.7 \%$ & 12 & $32.4 \%$ & \\
\hline Renal disorders & & & & & 0.772 \\
\hline No & 144 & $88.3 \%$ & 34 & $91.9 \%$ & \\
\hline Yes & 19 & $11.7 \%$ & 3 & $8.1 \%$ & \\
\hline Hematological disorders & & & & & 0.999 \\
\hline No & 152 & $93.3 \%$ & 35 & $94.6 \%$ & \\
\hline Yes & 11 & $6.7 \%$ & 2 & $5.4 \%$ & \\
\hline Neurological disorders & & & & & 0.120 \\
\hline No & 159 & $97.5 \%$ & 34 & $91.9 \%$ & \\
\hline Yes & 4 & $2.5 \%$ & 3 & $8.1 \%$ & \\
\hline Psychological disorders & & & & & $<0.001^{*}$ \\
\hline No & 158 & $96.9 \%$ & 29 & $78.4 \%$ & \\
\hline Yes & 5 & $3.1 \%$ & 8 & $21.6 \%$ & \\
\hline Immunological disorders & & & & & 0.616 \\
\hline No & 158 & $96.9 \%$ & 35 & $94.6 \%$ & \\
\hline Yes & 5 & $3.1 \%$ & 2 & $5.4 \%$ & \\
\hline Others chronic diseases & & & & & 0.235 \\
\hline No & 148 & $90.8 \%$ & 31 & $83.8 \%$ & \\
\hline Yes & 15 & $9.2 \%$ & 6 & $16.2 \%$ & \\
\hline
\end{tabular}

$* p$-value $\leq 0.05$ is considered significant.

There is a statistically significant difference between the severity of anxiety and psychological disorders $(P<0.001)$, but there was no significant difference between the severity of anxiety and immunological disorders, DM, HTN, CHD, renal disorders, hematological disorders, neurological disorders, and other chronic diseases. After adjusting for age, sex, education, and the presence of psychological disorders, Table 8 revealed that the existence of an existing psychiatric disorder was the only predictor linked with the occurrence of moderate to severe anxiety. The presence of a psychiatric disease, elevated the probability of occurrence by 9 times (OR, 95 percent CI for OR was $13.080,3.261$ to 52.464 ) (Table 8 ). 
Table 8 . Multivariable binary logistic regression analysis for prediction of moderate to severe anxiety from different risk factors

\begin{tabular}{|c|c|c|c|c|c|}
\hline \multirow{2}{*}{ Independent variables } & \multirow{2}{*}{ B } & \multirow{2}{*}{ p-value } & \multirow{2}{*}{$\begin{array}{l}\text { Odds Ratio } \\
\text { (OR) }\end{array}$} & \multicolumn{2}{|c|}{ 95\% CI for $O R$} \\
\hline & & & & Lower & Upper \\
\hline Old age $\geq 60$ years & -.341 & 0.519 & 0.711 & 0.252 & 2.006 \\
\hline Male sex & -.379 & 0.434 & 0.684 & 0.265 & 1.771 \\
\hline High education & .133 & 0.837 & 1.142 & .324 & 4.023 \\
\hline Presence of psychiatric disorder & 2.571 & $0.001 *$ & 13.080 & 3.261 & 52.464 \\
\hline
\end{tabular}

$*$ p-value $\leq 0.05$ is considered significant $C I=$ confidence interval.

Correlation Analysis between the PHQ-9 score, the GAD-7 score and age, There was a strong significant linear positive correlation between PHQ-9 score and GAD-7 score $(r=0.728 ; p<0.001)$ (Table 9).

Table 9. correlation between total PHQ-9 score, total GAD-7 scores and age

\begin{tabular}{llcc}
\hline & & Total PHQ-9 score & Total GAD-7 score \\
\hline Age & $r$ & 0.051 & 0.066 \\
& $P$-value & 0.469 & 0.351 \\
\hline Total PHQ-9 Score & $r$ & ---- & 0.728 \\
& $P$-value & & $<0.001^{*}$ \\
\hline
\end{tabular}

* $p$-value $\leq 0.05$ is considered significant, $r$ : Pearson correlation

PHQ-9: Patient Health Questionnaire-9; GAD-7: Generalized Anxiety Disorder-7.

\section{Discussion}

Anxiety and depression are two of the most common psychological disorders, and they frequently coexist with other psychological disorders, somatic disorders, and each other (Cosco, Doyle, Ward, \& McGee, 2012). Screening for anxiety and depression in outpatient clinics, where symptoms are often milder and earlier than those seen by mental health professionals (Goldberg et al., 2017). Understanding the prevalence of comorbid depressive and anxiety disorders in older adults is critical for theoretical conceptualizations, assessment and diagnostic classification, prevention, and interventions. Previous clinical sample studies revealed high levels of comorbidity and its link to the severity of symptoms and disabilities (King-Kallimanis, Gum, \& Kohn, 2009).

The prevalence of depression among the studied participants was moderate to severe in $21 \%$ of participants. This finding is consistent with a study finding from Saudi Arabia (22.1 percent) (Alamri, Bari, \& Ali, 2017), (22\%) from the United States are using the same tool. However, it is lower than in Iran (43.2 percent) and Greece (32.4 percent) (Manea, Gilbody, \& McMillan, 2015). Depressive symptoms are especially common in hospitalized elderly people, with rates ranging from 5.9 to 81 percent higher than in the general population (Alamri et al., 2017).

Furthermore, for the PHQ-9 depression severity assessment method, sociodemographic factors found to be significantly associated with depression in this population were a higher likelihood of having chronic immunological disease or psychiatric disorder, which supports previous findings from other studies (Zhong et al., 2010). Besides that, several studies have found that depressive symptoms are significantly higher in hospitalized geriatric patients with medical conditions such as heart disease, diabetes, osteoporosis, stroke, and dementia, complicating the task of detecting depression early in this population (Alamri et al., 2017). The underlying reasons include the illness itself and the high medical costs, as well as inadequate medical care and a poor doctor-patient relationship (Wang et al., 2017).

Moreover, women were affected by depression at a higher rate than men, which is consistent with previous findings that depression is more prevalent in women (Alamri et al., 2017). The severity of depression in the studied group is higher in those 60 years old or older, married, with a low to moderate educational level, with chronic diseases, and with two chronic diseases or more. However, we found that gender and age were not risk factors, 
which is consistent with some but not all previous studies (Zhong et al., 2010). Female gender and the presence of psychiatric disorders are significant predictors of depression.

In terms of anxiety, our findings show that those 60 years old or older, females, married status, low to moderate educational level, presence of chronic diseases, and those with two chronic diseases or more have higher anxiety severity. There is a statistically significant difference between the severity of anxiety and the presence of psychological disorders. The PHQ-9 score and the GAD-7 score had a strong significant linear positive correlation. A number of variables were suspected to be associated with comorbid anxiety disorders in late-life depression based on the literature review and were investigated for their associations. Female gender, a history of depressive disorder, and depression severity were all associated with anxiety disorder comorbidity. The explanation for this could be found in the emerging evidence for a common genetic predisposition to anxiety and depression, a theory that has long been investigated with the belief that genetic variation in personality traits could play a role in the development of both anxiety and depression (Suradom et al., 2019). The presence of a psychiatric disease was found to be a strong positive predictor of the occurrence of anxiety in our study.

Anxiety has been linked to dysregulation of the hypothalamic-pituitary-adrenal axis and inflammation, which can lead to poor health. A recent study of hospitalized patients found that people with anxiety disorders had more comorbid physical conditions than people who did not have anxiety disorders. Anxiety, on the other hand, could be a reaction to an underlying medical illness, and physical illness can exacerbate anxiety; the possibility of a bidirectional relationship between anxiety and physical health should not be ruled out. However, compelling evidence from prospective studies has shown that anxiety can indeed increase the risk of serious chronic conditions such as cancer and coronary heart disease (CHD) (Remes et al., 2018).

When looking into the links between mental disorders and health outcomes, early-onset or late-onset anxiety disorders, as well as psychiatric comorbidity, should be taken into account. A large study of over 1 million Swedish men followed for over 20 years found that early-onset mental disorders were associated with an increased risk of incident CHD. Anxiety disorders, such as GAD, are frequently comorbid with major depressive disorder (MDD), and psychiatric comorbidity has been linked to a lower quality of life, a worse prognosis, and a higher use of mental health services than pure forms of the disorder. Identifying clinical aspects such as early-onset or late-onset forms of the condition, episode chronicity and frequency, and comorbidity with MDD can thus lead to better clinical management and insight into groups at high risk for health-care utilization (Remes et al., 2018).

\section{Conclusion}

The current study found a high prevalence of depression and anxiety disorders among the participants we studied. Using validated semi-structured diagnostic interviews, we discovered that using PHQ-9 10 to estimate depression prevalence yields estimates of $21 \%$. There is a statistically significant difference between the severity of depression and psychological and immunological disorders. Almost 18.5 percent of study participants experienced moderate to severe anxiety. . There is a statistically significant difference between the severity of anxiety and the presence of psychological disorders.

\section{Recommendations}

Our findings indicate an urgent need for clinicians in Saudi Arabia to improve their ability to detect and treat depression and anxiety in chronically ill middle and late adults. Our findings reaffirm the importance of providing appropriate specialized interventions to promote the mental health of chronically ill elderly, with a focus on high-risk groups.

\section{Strength and limitations}

The fact that we investigated a significant number of previously reported variables with appropriate measurements is one of the study's strengths. To date, this is one of the few and most extensive studies on comorbid depression and anxiety disorders in middle and late adults in Saudi Arabia's Al-Qassim region.

This study did have some limitations. First, we collected data from middle and older adult patients who attended outpatient clinics at Alshifaa hospital in Al-Qassim region. Second, because this study was conducted on people aged 45 and up, it may not be applicable to younger age groups.

\section{Competing Interests Statement}

The authors declare that there are no competing or potential conflicts of interest.

\section{References}

Alamri, S. H., Bari, A. I., \& Ali, A. T. (2017). Depression and associated factors in hospitalized elderly: A 
cross-sectional study in a Saudi teaching hospital. Annals of Saudi Medicine, 37(2), 122-129. https://doi.org/10.5144/0256-4947.2017.122

Cosco, T. D., Doyle, F., Ward, M., \& McGee, H. (2012). Latent structure of the Hospital Anxiety And Depression Scale: A 10-year systematic review. Journal of Psychosomatic Research, 72(3), 180-184. https://doi.org/10.1016/j.jpsychores.2011.06.008

Goldberg, D. P., Reed, G. M., Robles, R., Minhas, F., Razzaque, B., Fortes, S., ... Saxena, S. (2017). Screening for anxiety, depression, and anxious depression in primary care: A field study for ICD-11 PHC. Journal of Affective Disorders, 213, 199-206. https://doi.org/10.1016/j.jad.2017.02.025

Guerrero J. M., \& Ramos, P. (2015). Mind Mapping for Reading and Understanding Scientific Literature. International Journal of Current Advanced Research, 4(11), 485-487. https://doi.org/10.1002/da

Hinz, A., Klein, A. M., Brähler, E., Glaesmer, H., Luck, T., Riedel-, S. G., ... Wirkner, K. (2016). Author' s Accepted Manuscript. Journal of Affective Disorders. https://doi.org/10.1016/j.jad.2016.12.012

Khoja, A. T., Aljawadi, M. H., Al-Shammari, S. A., Mohamed, A. G., Al-Manaa, H. A., Morlock, L., ... Khoja, T. A. M. (2018). The health of Saudi older adults; results from the Saudi National Survey for Elderly Health (SNSEH) 2006-2015. Saudi Pharmaceutical Journal, 26(2), 292-300. https://doi.org/10.1016/j.jsps.2017.11.008

King-Kallimanis, B., Gum, A. M., \& Kohn, R. (2009). Comorbidity of depressive and anxiety disorders for older Americans in the national comorbidity survey-replication. American Journal of Geriatric Psychiatry, 17(9), 782-792. https://doi.org/10.1097/JGP.0b013e3181ad4d17

Manea, L., Gilbody, S., \& McMillan, D. (2015). A diagnostic meta-analysis of the Patient Health Questionnaire-9 (PHQ-9) algorithm scoring method as a screen for depression. General Hospital Psychiatry, 37(1), 67-75. https://doi.org/10.1016/j.genhosppsych.2014.09.009

Remes, O., Wainwright, N., Surtees, P., Lafortune, L., Khaw, K. T., \& Brayne, C. (2018). Generalised anxiety disorder and hospital admissions: Findings from a large, population cohort study. BMJ Open, 8(10), 1-11. https://doi.org/10.1136/bmjopen-2017-018539

Reynolds, K., Pietrzak, R. H., El-Gabalawy, R., Mackenzie, C. S., \& Sareen, J. (2015). Prevalence of psychiatric disorders in U.S. older adults: Findings from a nationally representative survey. World Psychiatry, 14(1), 74-81. https://doi.org/10.1002/wps.20193

Schoevers, R. A., Deeg, D. J. H., Van Tilburg, W., \& Beekman, A. T. F. (2005). Depression and generalized anxiety disorder: Co-occurrence and longitudinal patterns in elderly patients. American Journal of Geriatric Psychiatry, 13(1), 31-39. https://doi.org/10.1097/00019442-200501000-00006

Shevlin, M., Mcbride, O., Murphy, J., Gibson Miller, J., Hartman, T. K., Levita, L., ... Bentall, R. P. (n.d.). Anxiety, depression, traumatic stress and COVID-19-related anxiety in the UK general population during the COVID-19 pandemic. https://doi.org/10.1192/bjo.2020.109

Suradom, C., Wongpakaran, N., Wongpakaran, T., Lerttrakarnnon, P., Jiraniramai, S., Taemeeyapradit, U., ... Arunpongpaisal, S. (2019). Prevalence and associated factors of comorbid anxiety disorders in late-life depression: Findings from geriatric tertiary outpatient settings. Neuropsychiatric Disease and Treatment, 15, 199-204. https://doi.org/10.2147/NDT.S184585

Thapa, D. K., Visentin, D. C., Kornhaber, R., \& Cleary, M. (2020). Prevalence and factors associated with depression, anxiety, and stress symptoms among older adults: A cross-sectional population-based study. Nursing and Health Sciences (Vol. 22). (S.1.): (s.n.). https://doi.org/10.1111/nhs.12783

Vink, D., Aartsen, M. J., \& Schoevers, R. A. (2008). Risk factors for anxiety and depression in the elderly: A review. Journal of Affective Disorders, 106(1-2), 29-44. https://doi.org/10.1016/j.jad.2007.06.005

Wang, J., Wu, X., Lai, W., Long, E., Zhang, X., Li, W., ... Lin, H. (2017). Prevalence of depression and depressive symptoms among outpatients: A systematic review and meta-analysis. BMJ Open, 7(8), 1-14. https://doi.org/10.1136/bmjopen-2017-017173

WHO. (2017). Mental health of older adults. Repéré à https://www.who.int/news-room/fact-sheets/detail/mental-health-of-older-adults

Zhong, B. liang, Chen, H. hui, Zhang, J. fang, Xu, H. ming, Zhou, C., Yang, F., ... Zhou, L. (2010). Prevalence, correlates and recognition of depression among inpatients of general hospitals in Wuhan, China. General 
Hospital Psychiatry, 32(3), 268-275. https://doi.org/10.1016/j.genhosppsych.2010.01.016

\section{Copyrights}

Copyright for this article is retained by the author(s), with first publication rights granted to the journal.

This is an open-access article distributed under the terms and conditions of the Creative Commons Attribution license (http://creativecommons.org/licenses/by/4.0/). 